\title{
Fixed point theory of the soft Meir-Keeler type contractive mappings on a complete soft metric space
}

\author{
Chi-Ming Chen ${ }^{1}$ and Ing-Jer Lin² ${ }^{*}$
}

\section{"Correspondence:}

ijlin@mail.nknu.edu.tw

${ }^{2}$ Department of Mathematics,

National Kaohsiung Normal

University, Kaohsiung, Taiwan

Full list of author information is

available at the end of the article

\begin{abstract}
In this paper, we introduce the notions of soft Meir-Keeler contractive mappings and weaker $\phi$-Meir-Keeler contractive mappings, and the purpose of this paper is to prove two theorems which assures the existence of fixed points for these two soft Meir-Keeler type contractive mappings on a soft metric space. Our results generalize and improve many recent fixed point results in the literature.
\end{abstract}

MSC: $47 \mathrm{H} 10 ; 54 \mathrm{C} 60 ; 54 \mathrm{H} 25 ; 55 \mathrm{M} 2 \mathrm{O}$

Keywords: fixed point; soft metric spaces; soft Meir-Keeler contractive mapping

\section{Introduction and preliminaries}

In 1999, Molodtsov [1] initiated a novel concept of soft set theory, is a new mathematical tool for dealing with uncertainties. Soft set is a parameterized general mathematical tool which deal with a collection of approximate descriptions of objects. In the recent, many researchers contribute many structure on soft set theory; (see, e.g., [2-4]). Further, Das and Samanta [5] introduced a different notion of soft metric space by using a different concept of soft point and investigated some basic properties of these spaces.

Next, we recall some basic definitions about soft sets and the results from the literature. In the sequel, we let $\mathbb{R}$ be a set of all real numbers, let $\mathbb{Z}$ be a set of all integers and $\mathbb{N}$ be a set of all natural numbers. We also let $\mathcal{U}$ be an initial universe, let $E$ be a set of parameters and let $2^{\mathcal{U}}$ the collection of all subsets of $\mathcal{U}$.

Definition 1 [1] Let $A$ be a nonempty subset of $E$. A soft set $(F, A)$ on $\mathcal{U}$ is a set of the form

$$
(F, A)=\{(F(p), p): p \in E\},
$$

where $F: A \rightarrow 2^{\mathcal{U}}$ is a set-valued map such that $F(p)=\phi$ for all $p \notin A$. Further, $F$ is called an approximate function of $(F, A)$, and the collection of all soft sets on $\mathcal{U}$ will be denoted by $\mathcal{S}(\mathcal{U})$.

Definition 2 [6] The intersection of two soft sets $\left(F_{1}, A\right)$ and $\left(F_{2}, B\right)$ on $\mathcal{U}$ is the soft set $\left(F_{3}, C\right)$ where $C=A \cap B$ and for each $p \in C, F_{3}(p)=F_{1}(p) \cap F_{2}(p)$. This is denoted by

$$
\left(F_{1}, A\right) \tilde{\cap}\left(F_{2}, B\right)=\left(F_{3}, C\right) .
$$

(c) 2015 Chen and Lin. This article is distributed under the terms of the Creative Commons Attribution 4.0 International License (http://creativecommons.org/licenses/by/4.0/), which permits unrestricted use, distribution, and reproduction in any medium, provided you give appropriate credit to the original author(s) and the source, provide a link to the Creative Commons license, and indicate if changes were made. 
Definition 3 [6] A soft set $(F, A)$ on $\mathcal{U}$ is said to be a null soft set denoted $\Phi$ if for all $p \in A$, $F(p)=\phi$.

Definition 4 [6] A soft set $(F, A)$ on $\mathcal{U}$ is said to be an absolute soft set denoted $\tilde{A}$ if for all $p \in A, F(p)=A$.

Definition 5 [6] The union of two soft sets $\left(F_{1}, A\right)$ and $\left(F_{2}, B\right)$ on $\mathcal{U}$ is the soft set $\left(F_{3}, C\right)$ where $C=A \cup B$ and for each $p \in C$,

$$
F_{3}(p)= \begin{cases}F_{1}(p), & \text { if } p \in A \backslash B ; \\ F_{2}(p), & \text { if } p \in B \backslash A ; \\ F_{1}(p) \cup F_{2}(p), & \text { if } p \in A \cap B .\end{cases}
$$

This relationship is denoted by $\left(F_{1}, A\right) \tilde{\cup}\left(F_{2}, B\right)=\left(F_{3}, C\right)$

In [7], the authors introduced the following notion of soft points.

Definition 6 [7] A soft set $(F, E)$ on $\mathcal{U}$ is said to be a soft point if there is exactly one $p \in E$ such that $F(p)=\{x\}$ for some $x \in \mathcal{U}$ and $F(q)=\phi$ for all $q \in \mathcal{U} \backslash\{p\}$. It will be denoted by $\tilde{x}_{p}$.

In [5], the authors introduced the following notion of soft real numbers.

Definition 7 [5] Let $B(\mathbb{R})$ be the collection of all nonempty bounded subsets of $\mathbb{R}$. Then the mapping $F: E \rightarrow B(\mathbb{R})$ is called a soft real set. If $(F, E)$ is a singleton soft set, then identifying $(F, E)$ with the corresponding soft element, it will be called a soft real number and denoted $\tilde{r}, \tilde{s}, \tilde{t}$, etc.

Let $\tilde{X}$ be the absolute soft set, where $(F, E)=\tilde{X}$, let $\mathcal{S P}(\tilde{X})$ be the collection of all soft points of $\tilde{X}$, and let $\mathbb{R}^{*}$ denote the set of all non-negative soft real numbers. In [5], the author introduced the notion of soft metric on the soft set $\tilde{X}$.

Definition 8 [5] A mapping $\tilde{d}: \mathcal{S P}(\tilde{X}) \times \mathcal{S P}(\tilde{X}) \rightarrow \mathbb{R}^{*}$ is said to be a soft metric on the soft set $\tilde{X}$ if $\tilde{d}$ satisfies the following conditions:

(M1) $\tilde{d}\left(\tilde{x}_{e_{1}}, \tilde{x}_{e_{2}}\right) \geq \overline{0}$, for all $\tilde{x}_{e_{1}}, \tilde{x}_{e_{2}} \in \tilde{X}$;

(M2) $\tilde{d}\left(\tilde{x}_{e_{1}}, \tilde{x}_{e_{2}}\right)=\overline{0}$ if and only if $\tilde{x}_{e_{1}}=\tilde{x}_{e_{2}}$;

(M3) $\tilde{d}\left(\tilde{x}_{e_{1}}, \tilde{x}_{e_{2}}\right)=\overline{0}$, for all $\tilde{x}_{e_{2}}, \tilde{x}_{e_{1}} \in \tilde{X}$;

(M4) $\tilde{d}\left(\tilde{x}_{e_{1}}, \tilde{x}_{e_{3}}\right) \tilde{\leq} \tilde{d}\left(\tilde{x}_{e_{1}}, \tilde{x}_{e_{2}}\right)+\tilde{d}\left(\tilde{x}_{e_{2}}, \tilde{x}_{e_{3}}\right)$, for all $\tilde{x}_{e_{1}}, \tilde{x}_{e_{2}}, \tilde{x}_{e_{3}} \in \tilde{X}$.

The soft set $\tilde{X}$ with a soft metric $\tilde{d}$ is called a soft metric space and denoted by $(\tilde{X}, \tilde{d}, E)$.

Definition 9 [5] Let $(\tilde{X}, \tilde{d}, E)$ be a soft metric space and $\tilde{\gamma}$ be a non-negative soft real number. Then $B\left(\tilde{x}_{e}, \tilde{\gamma}\right)=\left\{\tilde{y}_{e^{\prime}} \tilde{\in} \tilde{X}: \tilde{d}\left(\tilde{x}_{e}, \tilde{y}_{e^{\prime}}\right) \tilde{<} \tilde{\gamma}\right\}$ is called the soft open ball with center $\tilde{x}_{e}$ and radius $\tilde{\gamma}$, and $B\left[\tilde{x}_{e}, \tilde{\gamma}\right]=\left\{\tilde{y}_{e^{\prime}} \tilde{\in} \tilde{X}: \tilde{d}\left(\tilde{x}_{e}, \tilde{y}_{e^{\prime}}\right) \tilde{\leq} \tilde{\gamma}\right\}$ is called the soft closed ball with center $\tilde{x}_{e}$ and radius $\tilde{\gamma}$.

Definition 10 [5] Let $(\tilde{X}, \tilde{d}, E)$ be a soft metric space. Then the soft set $(F, E)$ is said to be soft open in $\tilde{X}$ with respect to $\tilde{d}$ if and only if all soft points of $(F, E)$ is interior points of $(F, E)$. 
Definition 11 [5] Let $\left\{\tilde{x}_{\lambda, n}\right\}_{n}$ be a sequence of soft points in a soft metric space $(\tilde{X}, \tilde{d}, E)$. Then the sequence $\left\{\tilde{x}_{\lambda, n}\right\}_{n}$ is said to be convergent in $(\tilde{X}, \tilde{d}, E)$ if there is a soft point $\tilde{y}_{\nu} \tilde{\in} \tilde{X}$ such that

$$
\lim _{n \rightarrow \infty} \tilde{d}\left(\tilde{x}_{\lambda, n}, \tilde{y}_{v}\right)=\overline{0}
$$

Definition 12 [5] Let $\left\{\tilde{x}_{\lambda, n}\right\}_{n}$ be a sequence of soft points in a soft metric space $(\tilde{X}, \tilde{d}, E)$. Then $\left\{\tilde{x}_{\lambda, n}\right\}_{n}$ is said to be a Cauchy sequence in $(\tilde{X}, \tilde{d}, E)$ if there is a soft point $\tilde{y}_{v} \tilde{\in} \tilde{X}$ such that

$$
\lim _{i, j \rightarrow \infty} \tilde{d}\left(\tilde{x}_{\lambda, i}, \tilde{x}_{\lambda, j}\right)=\overline{0}
$$

Definition 13 [5] A soft metric space $(\tilde{X}, \tilde{d}, E)$ is called complete if every Cauchy sequence in $\tilde{X}$ converges to some point of $\tilde{X}$.

\section{Fixed point theorem for the soft Meir-Keeler contractive mapping}

In this study, we also recall the Meir-Keeler contractive mapping [8].

Definition 14 [8] Let $(X, d)$ be a metric space, $f: X \rightarrow X$. Then $f$ is called a Meir-Keeler contractive mapping whenever for each $\eta>0$ there exists $\gamma>0$ such that for all $x, y \in X$,

$$
\eta \leq d(x, y)<\eta+\gamma \quad \Longrightarrow \quad d(f x, f y)<\eta \text {. }
$$

We introduce the new notion of a soft Meir-Keeler contractive mapping, as follows.

Definition 15 Let $(\tilde{X}, \tilde{d}, E)$ be a soft metric space and let $\varphi: E \rightarrow E$. A mapping $(f, \varphi)$ : $(\tilde{X}, \tilde{d}, E) \rightarrow(\tilde{X}, \tilde{d}, E)$ is called a soft Meir-Keeler contractive mapping if for any soft real number $\tilde{\eta}>\overline{0}$, there exists $\tilde{\gamma}>\overline{0}$ such that for each soft points $\tilde{x}_{\lambda}, \tilde{y}_{\mu} \in \mathcal{S P}(\tilde{X})$,

$$
\tilde{\eta} \tilde{\leq} \tilde{d}\left(\tilde{x}_{\lambda}, \tilde{y}_{\mu}\right) \tilde{<} \tilde{\eta}+\tilde{\gamma} \quad \Longrightarrow \quad \tilde{d}\left((f, \varphi)\left(\tilde{x}_{\lambda}\right),(f, \varphi)\left(\tilde{y}_{\mu}\right)\right) \tilde{<} \tilde{\eta} .
$$

Remark 1 Note that if $(f, \varphi)$ is a soft Meir-Keeler contractive mapping on $(\tilde{X}, \tilde{d}, E)$, then we have

$$
\tilde{d}\left((f, \varphi)\left(\tilde{x}_{\lambda}\right),(f, \varphi)\left(\tilde{y}_{\mu}\right)\right) \tilde{\leq} \tilde{d}\left(\tilde{x}_{\lambda}, \tilde{y}_{\mu}\right) .
$$

Further, if $\tilde{d}\left(\tilde{x}_{\lambda}, \tilde{y}_{\mu}\right)=\overline{0}$, then $\tilde{d}\left((f, \varphi)\left(\tilde{x}_{\lambda}\right),(f, \varphi)\left(\tilde{y}_{\mu}\right)\right)=\overline{0}$.

On the other hand, if $\tilde{d}\left(\tilde{x}_{\lambda}, \tilde{y}_{\mu}\right) \tilde{>} \overline{0}$, then

$$
\tilde{d}\left((f, \varphi)\left(\tilde{x}_{\lambda}\right),(f, \varphi)\left(\tilde{y}_{\mu}\right)\right) \tilde{<} \tilde{d}\left(\tilde{x}_{\lambda}, \tilde{y}_{\mu}\right) .
$$

Theorem 1 Let $(\tilde{X}, \tilde{d}, E)$ be a complete soft metric space, and let $(f, \varphi):(\tilde{X}, \tilde{d}, E) \rightarrow(\tilde{X}, \tilde{d}, E)$ be a soft Meir-Keeler contractive mapping on $(\tilde{X}, \tilde{d}, E)$. Then $(f, \varphi)$ has a unique fixed point, that is, there exists a unique soft point $\tilde{x}_{\lambda} \in \mathcal{S P}(\tilde{X})$ such that $(f, \varphi)\left(\tilde{x}_{\lambda}\right)=\tilde{x}_{\lambda}$.

Proof Let $\tilde{x}_{\lambda_{0}}^{0} \in \mathcal{S P}(\tilde{X})$ be given. Put

$$
\tilde{x}_{\lambda_{n+1}}^{n+1}=\left((f, \varphi)\left(\tilde{x}_{\lambda_{n}}^{n}\right)\right)=\left(f^{n+1}\left(\tilde{x}_{\lambda_{0}}^{0}\right)\right)_{\varphi^{n+1}\left(\lambda_{0}\right)},
$$


for each $n \in \mathbb{N} \cup\{0\}$. Then by the inequality (1), we have

$$
\begin{aligned}
\tilde{d}\left(\tilde{x}_{\lambda_{n}}^{n}, \tilde{x}_{\lambda_{n+1}}^{n+1}\right) & =\tilde{d}\left((f, \varphi)\left(\tilde{x}_{\lambda_{n-1}}^{n-1}\right),(f, \varphi)\left(\tilde{x}_{\lambda_{n}}^{n}\right)\right) \\
& \tilde{\alpha} \tilde{d}\left(\tilde{x}_{\lambda_{n-1}}^{n-1}, \tilde{x}_{\lambda_{n}}^{n}\right)
\end{aligned}
$$

for each $n \in \mathbb{N} \cup\{0\}$. Therefore the sequence $\left\{\tilde{d}\left(\tilde{x}_{\lambda_{n}}^{n}, \tilde{x}_{\lambda_{n+1}^{n+1}}^{n+1}\right)\right\}_{n}$ is decreasing, it must converge to some soft real number $\tilde{\eta} \geq \overline{0}$, that is, $\tilde{d}\left(\tilde{x}_{\lambda_{n}}^{n}, \tilde{x}_{\lambda_{n+1}^{n+1}}^{n+1}\right) \rightarrow \tilde{\eta}$, as $n \rightarrow \infty$. Notice that $\tilde{\xi}=\inf \left\{\tilde{d}\left(\tilde{x}_{\lambda_{n}}^{n}, \tilde{x}_{\lambda_{n+1}}^{n+1}\right): n \in \mathbb{N} \cup\{0\}\right\}$. We claim that $\tilde{\eta}=\overline{0}$. Suppose, on the contrary, that $\tilde{\eta} \tilde{>0}$. Since $f$ is a soft Meir-Keeler contractive mapping, corresponding to $\tilde{\eta}$, there exist $\tilde{\gamma}>\overline{0}$ and $k \in \mathbb{N}$ such that

$$
\begin{aligned}
& \tilde{\eta} \tilde{\leq} \tilde{d}\left(\tilde{x}_{\lambda_{k}}^{k}, \tilde{x}_{\lambda_{k+1}}^{k+1}\right) \tilde{<} \tilde{\eta}+\tilde{\gamma} \\
& \quad \Longrightarrow \quad \tilde{d}\left(\tilde{x}_{\lambda_{k+1}}^{k+1}, \tilde{x}_{\lambda_{k+2}}^{k+2}\right)=\tilde{d}\left((f, \varphi)\left(\tilde{x}_{\lambda_{k}}^{k}\right),(f, \varphi)\left(\tilde{x}_{\lambda_{k+1}}^{k+1}\right)\right) \tilde{<} \tilde{\eta} .
\end{aligned}
$$

This is a contradiction since $\tilde{\eta}=\inf \left\{\tilde{d}\left(\tilde{x}_{\lambda_{n}}^{n}, \tilde{x}_{\lambda_{n+1}}^{n+1}\right): n \in \mathbb{N} \cup\{0\}\right\}$. Thus, we obtain $\tilde{d}\left(\tilde{x}_{\lambda_{n}}^{n}\right.$, $\left.\tilde{x}_{\lambda_{n+1}^{n+1}}^{n+}\right) \rightarrow \overline{0}$, as $n \rightarrow \infty$.

We next show that $\left\{\tilde{x}_{\lambda_{n}}^{n}\right\}_{n}$ is a Cauchy sequence in $(\tilde{X}, \tilde{d}, E)$. To prove this, on the contrary, assume that a soft real number $\tilde{\epsilon} \tilde{>} \overline{0}$ such that for any $k \in \mathbb{N}$, there are $m_{k}, n_{k} \in \mathbb{N}$ with $n_{k}>m_{k} \geq k$ satisfying

$$
\tilde{d}\left(\tilde{x}_{\lambda_{m_{k}}}^{m_{k}}, \tilde{x}_{\lambda_{n_{k}}}^{n_{k}}\right) \tilde{\geq} \tilde{\epsilon}
$$

Further, corresponding to $m_{k} \geq k$, we can choose $n_{k}$ in such a way that it is the smallest integer with $n_{k}>m_{k} \geq k$ and $\tilde{d}\left(\tilde{x}_{\lambda_{m_{k}}}^{m_{k}}, \tilde{x}_{\lambda_{n_{k}}}^{n_{k}}\right) \tilde{\geq} \tilde{\epsilon}$. Therefore,

$$
\tilde{d}\left(\tilde{x}_{\lambda_{m_{k}}}^{m_{k}}, \tilde{x}_{\lambda_{n_{k}-2}}^{n_{k_{k}-2}}\right) \tilde{<} \tilde{\epsilon}
$$

Now we have, for all $k \in \mathbb{N}$,

$$
\begin{aligned}
& \tilde{\epsilon} \tilde{\leq} \tilde{d}\left(\tilde{x}_{\lambda_{m_{k}}}^{m_{k}}, \tilde{x}_{\lambda_{n_{k}}}^{n_{k}}\right) \\
& \tilde{\leq} \tilde{d}\left(\tilde{x}_{\lambda_{m_{k}}}^{m_{k}}, \tilde{x}_{\lambda_{n_{k}-2}}^{n_{k}-2}\right)+\tilde{d}\left(\tilde{x}_{\lambda_{n_{k}-2}}^{n_{k}-2}, \tilde{x}_{\lambda_{n_{k}-1}}^{n_{n_{k}-1}}\right)+\tilde{d}\left(\tilde{x}_{\lambda_{n_{k}-1}}^{n_{k}-1}, \tilde{x}_{\lambda_{n_{k}}}^{n_{k}}\right) \\
& \tilde{<} \tilde{\epsilon}+\tilde{d}\left(\tilde{x}_{\lambda_{n_{k}-2}}^{n_{k_{k}-2}}, \tilde{x}_{\lambda_{n_{k}-1}}^{n_{k}-1}\right)+\tilde{d}\left(\tilde{x}_{\lambda_{n_{k}-1}}^{n_{k}-1}, \tilde{x}_{\lambda_{n_{k}}}^{n_{k}}\right) \text {. }
\end{aligned}
$$

Letting $k \rightarrow \infty$ in the above inequality, we get

$$
\tilde{d}\left(\tilde{x}_{\lambda_{m_{k}}}^{m_{k}}, \tilde{x}_{\lambda_{n_{k}}}^{n_{k}}\right) \rightarrow \tilde{\epsilon} \quad \text { as } n \rightarrow \infty \text {. }
$$

On the other hand, we have

$$
\begin{aligned}
& \tilde{\epsilon} \tilde{\leq} \tilde{d}\left(\tilde{x}_{\lambda_{m_{k}}}^{m_{k}}, \tilde{x}_{\lambda_{n_{k}}}^{n_{k}}\right) \\
& \tilde{\leq} \tilde{d}\left(\tilde{x}_{\lambda_{m_{k}}}^{m_{k}}, \tilde{x}_{\lambda_{m_{k}+1}}^{m_{k}+1}\right)+\tilde{d}\left(\tilde{x}_{\lambda_{m_{k}+1}}^{m_{k}+1}, \tilde{x}_{\lambda_{n_{k}+1}}^{n_{k}+1}\right)+\tilde{d}\left(\tilde{x}_{\lambda_{n_{k}+1}}^{n_{k}+1}, \tilde{x}_{\lambda_{n_{k}}}^{n_{k}}\right) \\
& \tilde{\leq} \tilde{d}\left(\tilde{x}_{\lambda_{m_{k}}}^{m_{k}}, \tilde{x}_{\lambda_{m_{k}+1}}^{m_{k}+1}\right)+\tilde{d}\left(\tilde{x}_{\lambda_{m_{k}+1}}^{m_{k}+1}, \tilde{x}_{\lambda_{m_{k}}}^{m_{k}}\right)+\tilde{d}\left(\tilde{x}_{\lambda_{m_{k}}}^{m_{k}}, \tilde{x}_{\lambda_{n_{k}}}^{n_{k}}\right) \\
& +\tilde{d}\left(\tilde{x}_{\lambda_{n_{k}}}^{n_{k}}, \tilde{x}_{\lambda_{n_{k}+1}}^{n_{n_{k}+1}}\right)+\tilde{d}\left(\tilde{x}_{\lambda_{n_{k}+1}}^{n_{k}+1}, \tilde{x}_{\lambda_{n_{k}}}^{n_{k}}\right) \text {. }
\end{aligned}
$$


Letting $k \rightarrow \infty$ in the above inequality, we get

$$
\tilde{d}\left(\tilde{x}_{\lambda_{m_{k}+1}}^{m_{k+1}}, \tilde{x}_{\lambda_{n_{k}+1}}^{n_{k+1}}\right) \rightarrow \tilde{\epsilon} \quad \text { as } n \rightarrow \infty
$$

Since $f$ is soft Meir-Keeler contractive mapping, we have

$$
\begin{aligned}
\tilde{d}\left(\tilde{x}_{\lambda_{m_{k}+1}}^{m_{k}+1}, \tilde{x}_{\lambda_{n_{k}+1}}^{n_{k}+1}\right) & =\tilde{d}\left((f, \varphi)\left(\tilde{x}_{\lambda_{m_{k}}}^{m_{k}}\right),(f, \varphi)\left(\tilde{x}_{\lambda_{n_{k}}}^{n_{k}}\right)\right) \\
& \tilde{<} \tilde{d}\left(\tilde{x}_{\lambda_{m_{k}}}^{m_{k}}, \tilde{x}_{\lambda_{n_{k}}}^{n_{k}}\right) .
\end{aligned}
$$

Letting $k \rightarrow \infty$ in the above inequality, we get $\tilde{\epsilon} \tilde{<} \tilde{\epsilon}$, which implies a contradiction. Thus, $\left\{\tilde{x}_{\lambda_{n}}^{n}\right\}$ is a Cauchy sequence in $(\tilde{X}, \tilde{d}, E)$.

Since $(\tilde{X}, \tilde{d}, E)$ is complete, there exists $\tilde{x}_{\lambda}^{*} \in \tilde{X}$ such that

$$
\tilde{x}_{\lambda_{n}}^{n} \rightarrow \tilde{x}_{\lambda}^{*} \quad \text { as } n \rightarrow \infty
$$

that is,

$$
\tilde{d}\left(\tilde{x}_{\lambda_{n}}^{n}, \tilde{x}_{\lambda}^{*}\right) \rightarrow \overline{0} \quad \text { as } n \rightarrow \infty
$$

We also have

$$
\begin{aligned}
\tilde{d}\left((f, \varphi)\left(\tilde{x}_{\lambda}^{*}\right), \tilde{x}_{\lambda}^{*}\right) & \tilde{\leq} \tilde{d}\left((f, \varphi)\left(\tilde{x}_{\lambda_{n}}^{n}\right),(f, \varphi)\left(\tilde{x}_{\lambda}^{*}\right)\right)+\tilde{d}\left((f, \varphi)\left(\tilde{x}_{\lambda_{n}}^{n}\right), \tilde{x}_{\lambda}^{*}\right) \\
& \tilde{<} \tilde{d}\left(\tilde{x}_{\lambda_{n}}^{n}, \tilde{x}_{\lambda}^{*}\right)+\tilde{d}\left(\tilde{x}_{\lambda_{n+1}^{n+1}}^{n+1}, \tilde{x}_{\lambda}^{*}\right) .
\end{aligned}
$$

Letting $k \rightarrow \infty$ in the above inequality, we get

$$
\tilde{d}\left((f, \varphi)\left(\tilde{x}_{\lambda}^{*}\right), \tilde{x}_{\lambda}^{*}\right)=\overline{0} .
$$

This implies that $(f, \varphi)\left(\tilde{x}_{\lambda}^{*}\right)=\tilde{x}_{\lambda}^{*}$, and hence $\tilde{x}_{\lambda}^{*}$ is a fixed point of the mapping $(f, \varphi)$.

Finally, let $\tilde{y}_{\lambda}^{*}$ be another fixed point of the mapping $(f, \varphi)$. Then

$$
\tilde{d}\left(\tilde{y}_{\lambda}^{*}, \tilde{x}_{\lambda}^{*}\right)=\tilde{d}\left((f, \varphi)\left(\tilde{y}_{\lambda}^{*}\right),(f, \varphi)\left(\tilde{x}_{\lambda}^{*}\right)\right) \tilde{<} \tilde{d}\left(\tilde{y}_{\lambda}^{*}, \tilde{x}_{\lambda}^{*}\right),
$$

which implies a contradiction. Thus, $\tilde{x}_{\lambda}^{*}=\tilde{y}_{\lambda}^{*}$, that is, the fixed point of the mapping $(f, \varphi)$ is unique.

\section{Fixed point theorem for the soft weaker $\phi$-Meir-Keeler contractive mapping}

Definition 16 Let $\phi: \mathbb{R}^{*} \rightarrow \mathbb{R}^{*}$. Then $\phi$ is called a soft weaker Meir-Keeler mapping if for each soft real number $\tilde{\eta} \tilde{>} \overline{0}$, there exists a soft real number $\tilde{\gamma} \tilde{>} \overline{0}$ such that for each soft points $\tilde{t} \in \mathbb{R}^{*}$

$$
\tilde{\eta} \tilde{\leq} \tilde{t} \tilde{<} \tilde{\eta}+\tilde{\gamma} \quad \Longrightarrow \quad \exists n_{0} \in \mathbb{N}, \quad \phi^{n_{0}}(\tilde{t}) \tilde{<} \tilde{\eta} .
$$

In the sequel, we denote by $\Phi$ the class of functions $\phi: \mathbb{R}^{*} \rightarrow \mathbb{R}^{*}$ satisfying the following conditions: 
$\left(\phi_{1}\right) \phi$ is a nondecreasing soft weaker Meir-Keeler mapping with $\phi(\tilde{t})=\overline{0}$ iff $\tilde{t}=\overline{0}$;

$\left(\phi_{2}\right)\left\{\phi^{n}(\tilde{t})\right\}_{n \in \mathbb{N}}$ is decreasing for all $\tilde{t} \in \mathbb{R}^{*}$;

$\left(\phi_{3}\right)$ if $\lim _{n \rightarrow \infty} \tilde{t}=\gamma$, then $\lim _{n \rightarrow \infty} \phi^{n}(\tilde{t}) \tilde{\leq} \gamma$.

Example 1 Let $\phi: \mathbb{R}^{*} \rightarrow \mathbb{R}^{*}$ be defined by

$$
\phi(\tilde{t})=\frac{\widetilde{2}}{3} t
$$

Then $\phi$ is a soft weaker Meir-Keeler mapping and $\phi \in \Phi$.

We introduce the new notion of soft weaker $\phi$-Meir-Keeler contractive mapping, as follows.

Definition 17 Let $(\tilde{X}, \tilde{d}, E)$ be a soft metric space, and let $\phi \in \Phi$. A mapping $(f, \varphi)$ : $(\tilde{X}, \tilde{d}, E) \rightarrow(\tilde{X}, \tilde{d}, E)$ is called a soft weaker $\phi$-Meir-Keeler contractive mapping if, for any soft real number $\tilde{\eta}>\overline{0}$, there exists $\tilde{\gamma}>\overline{0}$ such that for each soft points $\tilde{x}_{\lambda}, \tilde{y}_{\mu} \in \mathcal{S P}(\tilde{X})$,

$$
\tilde{\eta} \tilde{\leq} \phi\left(\tilde{d}\left(\tilde{x}_{\lambda}, \tilde{y}_{\mu}\right)\right) \tilde{<} \tilde{\eta}+\tilde{\gamma} \quad \Longrightarrow \quad \tilde{d}\left((f, \varphi)\left(\tilde{x}_{\lambda}\right),(f, \varphi)\left(\tilde{y}_{\mu}\right)\right) \tilde{<} \tilde{\eta} .
$$

Remark 2 Note that if $(f, \varphi)$ is a soft weaker $\phi$-Meir-Keeler contractive mapping on $(\tilde{X}, \tilde{d}, E)$, then we have

$$
\tilde{d}\left((f, \varphi)\left(\tilde{x}_{\lambda}\right),(f, \varphi)\left(\tilde{y}_{\mu}\right)\right) \tilde{<} \phi\left(\tilde{d}\left(\tilde{x}_{\lambda}, \tilde{y}_{\mu}\right)\right) .
$$

Example 2 Let $(\tilde{\mathbb{R}}, \tilde{d}, E)$ be a soft metric space with the following metrics:

$$
\begin{aligned}
& d(x, y)=\max \{|x|,|y|\}, \quad d_{\varphi}(\lambda, \mu)=|\lambda-\mu| \quad \text { and } \\
& \tilde{d}\left(\tilde{x}_{\lambda}, \tilde{y}_{\mu}\right)=\frac{1}{4} d_{\varphi}(\lambda, \mu)+d(x, y),
\end{aligned}
$$

where $E=[1, \infty), \varphi(t)=\frac{1}{2}\left(t+\frac{1}{2 t}\right)$ for $t \in[1, \infty)$.

We next let $f(x)=\frac{1}{2} x$, and let $\phi: \mathbb{R}^{*} \rightarrow \mathbb{R}^{*}$ be defined by

$$
\phi(\tilde{t})=\frac{\widetilde{2}}{3} t
$$

Then

$$
\begin{aligned}
\tilde{d}\left((f, \varphi)\left(\tilde{x}_{\lambda}\right),(f, \varphi)\left(\tilde{y}_{\mu}\right)\right) & =\tilde{d}\left(\widetilde{\left(\frac{1}{2} x\right)_{\lambda+\frac{1}{2 \lambda}}}, \widetilde{\left(\frac{1}{2} y\right)_{\mu+\frac{1}{2 \mu}}}\right) \\
& =\frac{1}{4}\left|\frac{1}{2}\left(\lambda+\frac{1}{2 \lambda}-\mu-\frac{1}{2 \mu}\right)\right|+\max \left\{\frac{1}{2}|x|, \frac{1}{2}|y|\right\} \\
& =\frac{1}{4}\left|\frac{1}{2}(\lambda-\mu)\left(1-\frac{1}{2 \lambda \mu}\right)\right|+\max \left\{\frac{1}{2}|x|, \frac{1}{2}|y|\right\} \\
& \tilde{\leq} \frac{1}{8}|\lambda-\mu|+\max \left\{\frac{1}{2}|x|, \frac{1}{2}|y|\right\}
\end{aligned}
$$




$$
\begin{aligned}
& \tilde{\leq} \frac{1}{6}|\lambda-\mu|+\frac{2}{3} \max \{|x|,|y|\} \\
& =\frac{2}{3}\left[\frac{1}{4}|\lambda-\mu|+\max \{|x|,|y|\}\right] \\
& =\frac{2}{3}\left[\frac{1}{4} d_{\varphi}(\lambda, \mu)+d(x, y)\right] \\
& =\phi\left(\tilde{d}\left(\tilde{x}_{\lambda}, \tilde{y}_{\mu}\right)\right) .
\end{aligned}
$$

Thus, $(f, \varphi)$ is a soft weaker $\phi$-Meir-Keeler contractive mapping on the soft metric space $(\tilde{\mathbb{R}}, \tilde{d}, E)$.

Theorem 2 Let $(\tilde{X}, \tilde{d}, E)$ be a complete soft metric space, and let $(f, \varphi):(\tilde{X}, \tilde{d}, E) \rightarrow(\tilde{X}, \tilde{d}, E)$ be a soft weaker $\phi$-Meir-Keeler contractive mapping on $(\tilde{X}, \tilde{d}, E)$. Then $(f, \varphi)$ has a fixed point, that is, there exists a soft point $\tilde{x}_{\lambda} \in \mathcal{S P}(\tilde{X})$ such that $(f, \varphi)\left(\tilde{x}_{\lambda}\right)=\tilde{x}_{\lambda}$.

Proof Let $\tilde{x}_{\lambda_{0}}^{0} \in \mathcal{S P}(\tilde{X})$ be given. Put

$$
\tilde{x}_{\lambda_{n+1}}^{n+1}=\left((f, \varphi)\left(\tilde{x}_{\lambda_{n}}^{n}\right)\right)=\left(f^{n+1}\left(\tilde{x}_{\lambda_{0}}^{0}\right)\right)_{\varphi^{n+1}\left(\lambda_{0}\right)},
$$

for each $n \in \mathbb{N} \cup\{0\}$. Then by the inequality (2), we have

$$
\begin{aligned}
\tilde{d}\left(\tilde{x}_{\lambda_{n}}^{n}, \tilde{x}_{\lambda_{n+1}}^{n+1}\right) & =\tilde{d}\left((f, \varphi)\left(\tilde{x}_{\lambda_{n-1}}^{n-1}\right),(f, \varphi)\left(\tilde{x}_{\lambda_{n}}^{n}\right)\right) \\
& \tilde{<\phi}\left(\tilde{d}\left(\tilde{x}_{\lambda_{n-1}}^{n-1}, \tilde{x}_{\lambda_{n}}^{n}\right)\right) \\
& =\phi\left(\tilde{d}\left((f, \varphi)\left(\tilde{x}_{\lambda_{n-2}}^{n-2}\right),(f, \varphi)\left(\tilde{x}_{\lambda_{n-1}}^{n-1}\right)\right)\right) \\
& \approx \phi\left(\phi\left(\tilde{x}_{\lambda_{n-2}}^{n-2}, \tilde{x}_{\lambda_{n-1}}^{n-1}\right)\right) \\
& \approx \cdots \\
& \approx \phi^{n}\left(\tilde{d}\left(\tilde{x}_{\lambda_{0}}^{0}, \tilde{x}_{\lambda_{1}}^{1}\right)\right) .
\end{aligned}
$$

Since $\left\{\phi^{n}\left(\tilde{d}\left(\tilde{x}_{\lambda_{0}}^{0}, \tilde{x}_{\lambda_{1}}^{1}\right)\right)\right\}_{n \in \mathbb{N}}$ is decreasing, it must converge to some $\tilde{\eta} \tilde{\geq} \overline{0}$. We claim that $\tilde{\eta}=\overline{0}$. On the contrary, assume that $\tilde{\eta} \tilde{>} \overline{0}$. Corresponds to this $\tilde{\eta}$, since $\phi$ is a weaker MeirKeeler mapping, there exists a soft real number $\tilde{\gamma} \sim \overline{0}$ such that

$$
\tilde{\eta} \tilde{\leq} \tilde{d}\left(\tilde{x}_{\lambda_{0}}^{0}, \tilde{x}_{\lambda_{1}}^{1}\right) \tilde{<} \tilde{\eta}+\tilde{\gamma} \quad \Longrightarrow \quad \exists n_{0} \in \mathbb{N}, \quad \phi^{n_{0}}\left(\tilde{d}\left(\tilde{x}_{\lambda_{0}}^{0}, \tilde{x}_{\lambda_{1}}^{1}\right)\right) \tilde{<} \tilde{\eta} .
$$

Since $\phi^{n}\left(\tilde{d}\left(\tilde{x}_{\lambda_{0}}^{0}, \tilde{x}_{\lambda_{1}}^{1}\right)\right) \rightarrow \tilde{\eta}$ as $n \rightarrow \infty$, there exists $m_{0} \in \mathbb{N}$ such that

$$
\tilde{\eta} \tilde{\leq} \phi^{m}\left(\tilde{d}\left(\tilde{x}_{\lambda_{0}}^{0}, \tilde{x}_{\lambda_{1}}^{1}\right)\right) \tilde{<} \tilde{\eta}+\tilde{\gamma}
$$

for all $m \geq m_{0}$. We also have

$$
\phi^{m_{0}+n_{0}}\left(\tilde{d}\left(\tilde{x}_{\lambda_{0}}^{0}, \tilde{x}_{\lambda_{1}}^{1}\right)\right) \tilde{<} \tilde{\eta} .
$$

So we get a contradiction. Therefore

$$
\phi^{n}\left(\tilde{d}\left(\tilde{x}_{\lambda_{0}}^{0}, \tilde{x}_{\lambda_{1}}^{1}\right)\right) \rightarrow \overline{0} \quad \text { as } n \rightarrow \infty,
$$


that is,

$$
\tilde{d}\left(\tilde{x}_{\lambda_{n}}^{n}, \tilde{x}_{\lambda_{n+1}}^{n+1}\right) \rightarrow \overline{0} \quad \text { as } n \rightarrow \infty
$$

We next show that $\left\{\tilde{x}_{\lambda_{n}}^{n}\right\}_{n}$ is a Cauchy sequence in $(\tilde{X}, \tilde{d}, E)$. To prove this, on the contrary, assume that a soft real number $\tilde{\epsilon} \tilde{>} \overline{0}$ such that for any $k \in \mathbb{N}$, there are $m_{k}, n_{k} \in \mathbb{N}$ with $n_{k}>m_{k} \geq k$ satisfying

$$
\tilde{d}\left(\tilde{x}_{\lambda_{m_{k}}}^{m_{k}}, \tilde{x}_{\lambda_{n_{k}}}^{n_{k}}\right) \tilde{\geq} \tilde{\epsilon}
$$

Further, corresponding to $m_{k} \geq k$, we can choose $n_{k}$ in such a way that it is the smallest integer with $n_{k}>m_{k} \geq k$ and $\tilde{d}\left(\tilde{x}_{\lambda_{m_{k}}}^{m_{k}}, \tilde{x}_{\lambda_{n_{k}}}^{n_{k}}\right) \tilde{\geq} \tilde{\epsilon}$. Therefore,

$$
\tilde{d}\left(\tilde{x}_{\lambda_{m_{k}}}^{m_{k}}, \tilde{x}_{\lambda_{n_{k}-2}}^{n_{k}-2}\right) \tilde{<} \tilde{\epsilon}
$$

Now we have, for all $k \in \mathbb{N}$,

$$
\begin{aligned}
& \tilde{\epsilon} \tilde{\leq} \tilde{d}\left(\tilde{x}_{\lambda_{m_{k}}}^{m_{k}}, \tilde{x}_{\lambda_{n_{k}}}^{n_{k}}\right) \\
& \tilde{\leq} \tilde{d}\left(\tilde{x}_{\lambda_{m_{k}}}^{m_{k}}, \tilde{x}_{\lambda_{n_{k}-2}}^{n_{k_{k}-2}}\right)+\tilde{d}\left(\tilde{x}_{\lambda_{n_{k}-2}}^{n_{k}-2}, \tilde{x}_{\lambda_{n_{k}-1}}^{n_{k-1}}\right)+\tilde{d}\left(\tilde{x}_{\lambda_{n_{k}-1}}^{n_{k}-1}, \tilde{x}_{\lambda_{n_{k}}}^{n_{k}}\right) \\
& \tilde{<} \tilde{\epsilon}+\tilde{d}\left(\tilde{x}_{\lambda_{n_{k}-2}}^{n_{k-2}}, \tilde{x}_{\lambda_{n_{k}-1}}^{n_{k}-1}\right)+\tilde{d}\left(\tilde{x}_{\lambda_{n_{k}-1}}^{n_{k}-1}, \tilde{x}_{\lambda_{n_{k}}}^{n_{k}}\right) \text {. }
\end{aligned}
$$

Letting $k \rightarrow \infty$ in the above inequality, we get

$$
\tilde{d}\left(\tilde{x}_{\lambda_{m_{k}}}^{m_{k}}, \tilde{x}_{\lambda_{n_{k}}}^{n_{k}}\right) \rightarrow \tilde{\epsilon} \quad \text { as } n \rightarrow \infty
$$

On the other hand, we have

$$
\begin{aligned}
& \tilde{\epsilon} \tilde{\leq} \tilde{d}\left(\tilde{x}_{\lambda_{m_{k}}}^{m_{k}}, \tilde{x}_{\lambda_{n_{k}}}^{n_{k}}\right) \\
& \tilde{\leq} \tilde{d}\left(\tilde{x}_{\lambda_{m_{k}}}^{m_{k}}, \tilde{x}_{\lambda_{m_{k}+1}}^{m_{k}+1}\right)+\tilde{d}\left(\tilde{x}_{\lambda_{m_{k}+1}}^{m_{k+1}}, \tilde{x}_{\lambda_{n_{k}+1}}^{n_{k}+1}\right)+\tilde{d}\left(\tilde{x}_{\lambda_{n_{k}+1}}^{n_{k}+1}, \tilde{x}_{\lambda_{n_{k}}}^{n_{k_{k}}}\right) \\
& \tilde{\leq} \tilde{d}\left(\tilde{x}_{\lambda_{m_{k}}}^{m_{k}}, \tilde{x}_{\lambda_{m_{k}+1}}^{m_{k}+1}\right)+\tilde{d}\left(\tilde{x}_{\lambda_{m_{k}+1}}^{m_{k}+1}, \tilde{x}_{\lambda_{m_{k}}}^{m_{k}}\right)+\tilde{d}\left(\tilde{x}_{\lambda_{m_{k}}}^{m_{k}}, \tilde{x}_{\lambda_{n_{k}}}^{n_{k}}\right) \\
& +\tilde{d}\left(\tilde{x}_{\lambda_{n_{k}}}^{n_{k}}, \tilde{x}_{\lambda_{n_{k}+1}}^{n_{k}+1}\right)+\tilde{d}\left(\tilde{x}_{\lambda_{n_{k}+1}}^{n_{k+1}+1}, \tilde{x}_{\lambda_{n_{k}}}^{n_{k}}\right) .
\end{aligned}
$$

Letting $k \rightarrow \infty$ in the above inequality, we get

$$
\tilde{d}\left(\tilde{x}_{\lambda_{m_{k}+1}}^{m_{k}+1}, \tilde{x}_{\lambda_{n_{k}+1}}^{n_{k}+1}\right) \rightarrow \tilde{\epsilon} \quad \text { as } n \rightarrow \infty .
$$

Since $f$ is soft weaker $\phi$-Meir-Keeler contractive mapping, we have

$$
\begin{aligned}
\tilde{d}\left(\tilde{x}_{\lambda_{m_{k}+1}}^{m_{k}+1}, \tilde{x}_{\lambda_{n_{k}+1}}^{n_{k}+1}\right) & =\tilde{d}\left((f, \varphi)\left(\tilde{x}_{\lambda_{m_{k}}}^{m_{k}}\right),(f, \varphi)\left(\tilde{x}_{\lambda_{n_{k}}}^{n_{k}}\right)\right) \\
& \tilde{<} \phi\left(\tilde{d}\left(\tilde{x}_{\lambda_{m_{k}}}^{m_{k}}, \tilde{x}_{\lambda_{n_{k}}}^{n_{k}}\right)\right) .
\end{aligned}
$$

By the condition $\left(\phi_{3}\right)$ of the function $\phi$ and letting $n \rightarrow \infty$, we obtain $\tilde{\epsilon} \tilde{<} \tilde{\epsilon}$, which implies a contradiction. Thus, $\left\{\tilde{x}_{\lambda_{n}}^{n}\right\}$ is a Cauchy sequence in $(\tilde{X}, \tilde{d}, E)$. 
Since $(\tilde{X}, \tilde{d}, E)$ is complete, there exists $\tilde{x}_{\lambda}^{*} \in \tilde{X}$ such that

$$
\tilde{x}_{\lambda_{n}}^{n} \rightarrow \tilde{x}_{\lambda}^{*} \text { as } n \rightarrow \infty
$$

that is,

$$
\tilde{d}\left(\tilde{x}_{\lambda_{n}}^{n}, \tilde{x}_{\lambda}^{*}\right) \rightarrow \overline{0} \quad \text { as } n \rightarrow \infty .
$$

And, we also have

$$
\begin{aligned}
\tilde{d}\left((f, \varphi)\left(\tilde{x}_{\lambda}^{*}\right), \tilde{x}_{\lambda}^{*}\right) & \tilde{\leq} \tilde{d}\left((f, \varphi)\left(\tilde{x}_{\lambda_{n}}^{n}\right),(f, \varphi)\left(\tilde{x}_{\lambda}^{*}\right)\right)+\tilde{d}\left((f, \varphi)\left(\tilde{x}_{\lambda_{n}}^{n}\right), \tilde{x}_{\lambda}^{*}\right) \\
& \tilde{<} \tilde{d}\left(\tilde{x}_{\lambda_{n}}^{n}, \tilde{x}_{\lambda}^{*}\right)+\tilde{d}\left(\tilde{x}_{\lambda_{n+1}}^{n+1}, \tilde{x}_{\lambda}^{*}\right) .
\end{aligned}
$$

Letting $k \rightarrow \infty$ in the above inequality, we get

$$
\tilde{d}\left((f, \varphi)\left(\tilde{x}_{\lambda}^{*}\right), \tilde{x}_{\lambda}^{*}\right)=\overline{0} .
$$

This implies that $(f, \varphi)\left(\tilde{x}_{\lambda}^{*}\right)=\tilde{x}_{\lambda}^{*}$, and hence $\tilde{x}_{\lambda}^{*}$ is a fixed point of the mapping $(f, \varphi)$.

\section{Competing interests}

The authors declare that they have no competing interests.

\section{Authors' contributions}

Both authors contributed equally and significantly in writing this paper. Both authors read and approved the final manuscript.

\section{Author details}

'Department of Applied Mathematics, National Hsinchu University of Education, Hsinchu, Taiwan. ${ }^{2}$ Department of Mathematics, National Kaohsiung Normal University, Kaohsiung, Taiwan.

\section{Acknowledgements}

This research was supported by the Ministry of Science and Technology of the Republic of China.

Received: 5 March 2015 Accepted: 8 May 2015 Published online: 10 June 2015

\section{References}

1. Molodtsov, DA: Soft set theory - first results. Comput. Math. Appl. 37, 19-31 (1999)

2. Ali, Ml, Feng, F, Liu, XY, Min, WK, Shabir, M: On some new operations in soft set theory. Comput. Math. Appl. 62, 351-358 (2011)

3. Chen, D: The parameterization reduction of soft sets and its applications. Comput. Math. Appl. 49, 757-763 (2005)

4. Babitha, KV, Suntil, JJ: Soft set relations and functions. Comput. Math. Appl. 60, 1840-1849 (2010)

5. Das, S, Samanta, SK: Soft real sets, soft real numbers and their properties. J. Fuzzy Math. 20(3), 551-576 (2012)

6. Maji, PK, Biswas, R, Roy, AR: Soft set theory. Comput. Math. Appl. 45, 555-562 (2003)

7. Bayramov, S, Gunduz, C: Soft locally compact and soft paracompact spaces. J. Math. Syst. Sci. 3, 122-130 (2013)

8. Meir, A, Keeler, E: A theorem on contraction mappings. J. Math. Anal. Appl. 28, 326-329 (1969) 